\title{
Modified Goel's Methods for Basilar Impression: A Case Report with Literature
}

\author{
Shunji Asamoto, ${ }_{1}^{1}$ Yasuyuki Fukui, ${ }^{1}$ Makoto Nishiyama, ${ }_{1}^{1}$ Masayuki Ishikawa, ${ }^{1}$ Satoshi Nakamura, \\ Masaki Nagashima, ${ }^{1}$ Jun Muto, ${ }^{1}$ and Hiroyuki Jimbo, ${ }^{1,2}$
}

We report the case of a 57-year-old woman who had basilar impression manifesting as severe myelopathy and occipital neuralgia and was treated by distraction and fixation performed using a modification of Goel's method. Magnetic resonance imaging (MRI) and computed tomography (CT) scans showed severe myelocompression by the dens of the axis from the ventral side and occipitalization of the atlas. After traction using a Halo vest, C1-2 facet distraction and fixation was performed in one stage using a modified Goel's method. Although Goel et al. used a custom-made spacer to distract the facet joints, we used a threaded titanium cylindrical cage that was inserted into the joint to fix the C1-2 facet joint with posterior fixation from occipital bone to C5. Postoperatively, gradual symptomatic and neurological amelioration were observed. The atlantoaxial joints were bone-fused at 3 years post-operation. Distraction and fixation performed using this modified version of Goel's method was effective for treating basilar invagination. The threaded titanium cylindrical cage provided adequate C1-2 space and strong initial fixation.

Keywords: basilar impression, modified Goel's method, threaded titanium cylindrical cage

\section{Introduction}

Basilar impression (BI) is defined as prolapse of the cervical spine (dens) into the skull base and is classified into Types A and B. ${ }^{1)}$ Several surgical options are available for BI. Posterior decompression and fixation are typically performed first, with a trans-oral approach selected second. Goel et al. recently presented a surgical technique for BI and rheumatoid disease that involves distraction and fixation of the $\mathrm{C} 1-2$ facet joint. ${ }^{2-4)}$

Here, we present a case of BI that was treated with distraction and fixation using a modified version of Goel's original technique.

\section{Case Report}

A 57-year-old woman had a 3-year history of occipitalgia. Upon examination, the patient showed occipitalgia, poor

\footnotetext{
${ }^{1}$ Spine and Spinal Cord Center, Mita Hospital, International University of Health and Welfare, Tokyo

${ }^{2}$ Department of Neurosurgery, Tokyo Medical University Hachioji Medical Center, Hachioji, Tokyo
}

Received: February 27, 2015; Accepted: August 26, 2015 dexterity and coordination of both hands, and ataxic and spastic gait associated with severe myelopathy and numbness of both the forearms and hands. The Japanese orthopedic score (JOA) was 5. Preoperative plain radiography and computed tomography (CT) scans revealed $\mathrm{C} 1$ assimilation and BI of the dens (Fig. 1A) and significant myelocompression by the $\mathrm{C} 2$ odontoid process (Fig. 1B).

\section{Treatment}

First, the patient underwent traction with $2 \mathrm{~kg}$ weight but this was discontinued because of severe neck pain. Therefore, traction was performed gently using a Halo vest (Fig. 2A). ${ }^{3)}$ The patient was pulled by the gantry of the Halo vest by $1 \mathrm{~cm} /$ week for 4 weeks. The occipitalgia ameliorated immediately, and numbness gradually reduced with traction.

After the traction, CT scans revealed marked reduction of the odontoid process on the caudal side (Fig. 2B, C). The patient then underwent surgery to avoid re-invagination in a prone position using a modification of Goel's method. Bilateral atlantoaxial facet joints were exposed after cutting the $\mathrm{C} 2$ ganglion. Using a microscope, we determined the articular surfaces to be "bone-to-bone" and observed marked degeneration of joints similar to degenerative lumbar listhesis. The facets were meticulously distracted using an appropriately sized shaver. Goel et al. used a custom-made spacer to distract the facet joints, ${ }^{3)}$ but we used a 6-mm diameter threaded titanium
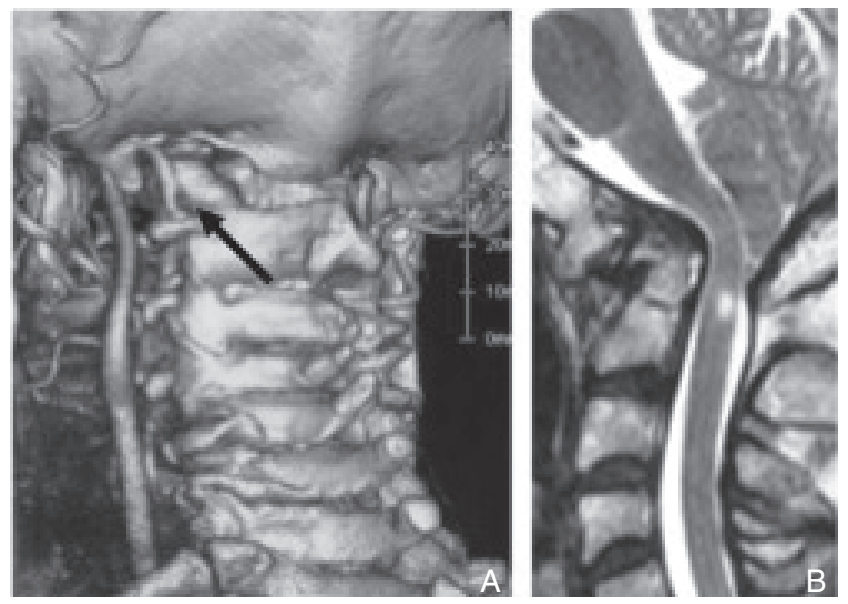

Fig. 1 A: Preoperative computed tomography (CT) scans with angiography revealing $\mathrm{Cl}$ assimilation of the atlas and hypoplasia of vertebral artery of left side (arrow). B: Preoperative sagittal magnetic resonance imaging (MRI) $\mathrm{T}_{2}$-weighted showing ventral myelocompression. 

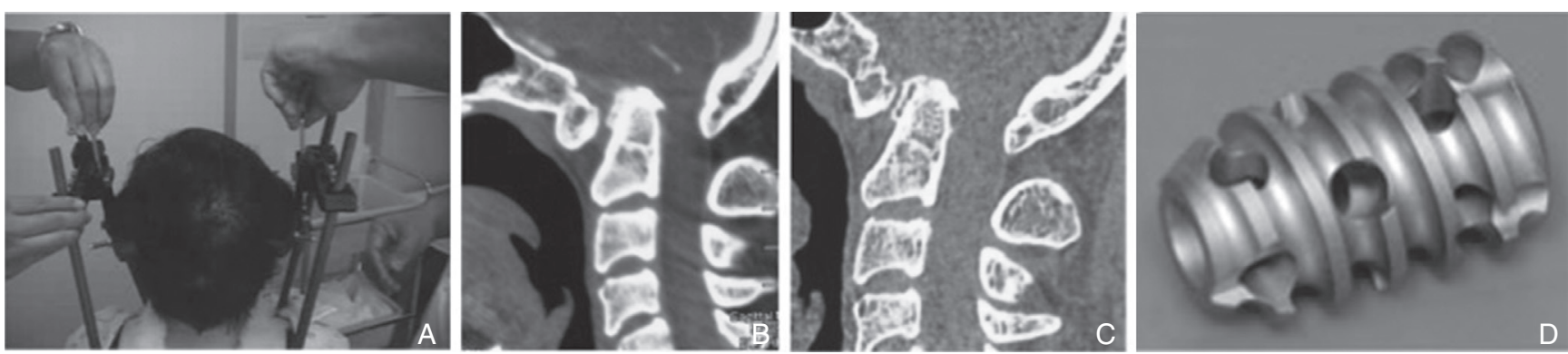

Fig. 2 A: Photograph showing lift of the Halo vest gantry, B: Pre-traction, C: post-traction computed tomography scans revealing marked reduction of the odontoid process, and D: the m-cage ${ }^{\circledR}$.
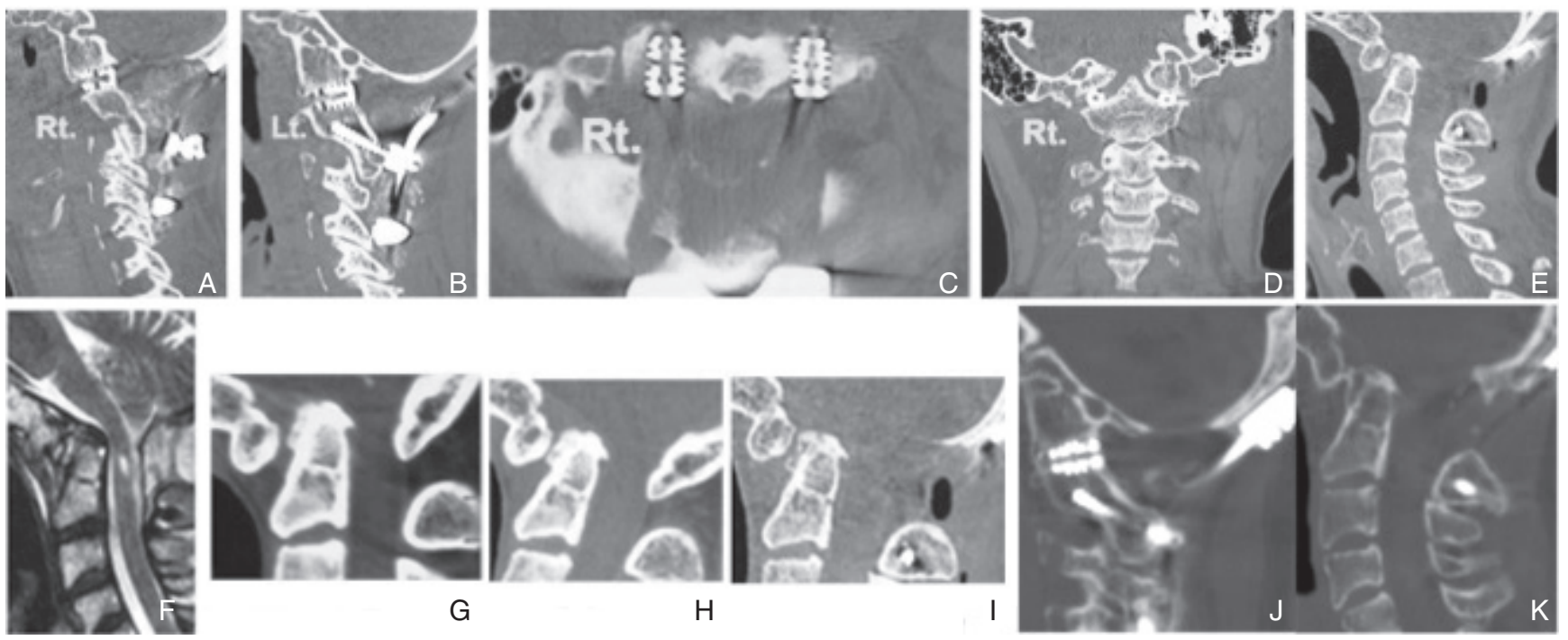

Fig. 3 Postoperative right view (A), left view (B), coronal views (C, D), sagittal view (E) of computed tomography (CT) scans and magnetic resonance imaging (MRI) $\mathrm{T}_{2}$-weighted $(\mathrm{F})$ showing reduced odontoid process of the axis. CT scans showing position of the dens at pre-treatment (G), post-traction $(\mathrm{H})$, and post-operative (I) time points. CT scans taken 3 years after the surgery shows $\mathrm{C} 1-2$ facet fusion and no recurrence of basilar impression (J, K).

cylindrical cage (Fig. 2D; m-cage ${ }^{\circledR}$; Ammtec Inc., Tokyo) on each side. Local bones were filled in around the cages. This cage was inserted into the joint smoothly with natural distraction. During the manipulation of the $\mathrm{C} 1-2$ facet joints, vertebral arteries were not identified and not necessary to see. Rod-and-screw fixation was conducted from the occipital bone to $\mathrm{C} 5$ using the VERTEX MAX ${ }^{\circledR}$ reconstruction system (Medtronic Sofamor Danek, Memphis, Tennessee, USA). We used a pedicle screw on the left side of $\mathrm{C} 2$, a laminar screw on the right side of $\mathrm{C} 2$, and applied a bilateral transarticular screw on $\mathrm{C} 4$. We expected to fuse solidly by fixing from the occipital bone to C5 (Fig. 3A-I). In this procedure, the threaded titanium cylindrical cage (m-cage $\left.{ }^{\circledR}\right)$ was inserted into $\mathrm{C} 1-2$ facet joint. There may be higher risk to cause sinking in the future, if only stand alone was done. As the original procedure done by Goel et al., the posterior fixation by the rod-and screw system was necessary to prevent the sinking.

Somatosensory-evoked potentials and transcranial motorevoked potentials could not detect any damage during the surgery.

\section{Outcome}

After the surgery, gradual symptomatic and neurological amelioration were observed. The JOA score improved to 13. The gait, dexterity, and incoordination of hands improved. The atlantoaxial joints were considered to be bone-fused on C1-2 facet, and no recurrence of BI was noticed on CT scans performed 3 years post-operatively (Fig. 3J, K).

\section{Discussion}

We report the first case of BI treated using a modification of Goel's method. In our procedure, for C1-2 facet joint distraction, a threaded titanium cylindrical cage was inserted to fix the $\mathrm{C} 1-2$ facet joint with posterior fixation from occipital bone to $\mathrm{C} 5$ using the reconstruction system (VERTEX $\left.M X^{\circledR}\right)$. Our method differed from Goel's in that Goel et al. used an original spacer to distract $\mathrm{C} 1-2$ space before fixation, while we used a thread-type cage to fix the $\mathrm{C} 1-2$ facet joint. Both performed posterior fixation with rod-and-screw system. Our case report proves that this modification enables a stable fixation. 
Previous reports have described different surgical approaches for similar diseases: ${ }^{1-7)}$ however, two important points must be highlighted. Instability associated with the assimilated arch of $\mathrm{C} 1$ led to relative $\mathrm{BI}$, causing $\mathrm{C} 1-2$ instability. We believe that the $\mathrm{C} 1-2$ joint causes listhesis, resulting in BI. In this case, CT scans showed bilateral C1-2 facet joints with a slant and listhesis. If the occipital neuralgia had been caused by instability and BI was accompanied by myelopathy, both fixation and decompression could be considered necessary. In the present case, occipitalgia and numbness in the hands ameliorated gradually after wearing the Halo vest. When considered as a one-stage operation, Goel's method, ${ }^{2)}$ Tuere's operation, ${ }^{4)}$ or cervical traction and occipitocervical fixation ${ }^{1)}$ seemed to represent the ideal procedures for this case. We chose Goel's method due to easy orientation with the posterior approach and insertion of the spacer into the facet joint of $\mathrm{C} 1-2$ without taking load against the $\mathrm{C} 2$ pedicle screw or articular screws. Although no detailed description of the inter-space distance of the distracted $\mathrm{C} 1-2$ facet joint was provided in Botelho's article, ${ }^{1)}$ the dynamic load against each screw is expected to be sufficient for $\mathrm{C} 1-2$ facet destruction and fixation. Even if $\mathrm{BI}$ is easily reduced by traction, ${ }^{1)}$ maintaining separation of the $\mathrm{C} 1-2$ joint is not easy. In this case using $\mathrm{m}$-cage achieved the initial fixation firmly with the smooth distraction. The reason could be m-cage we used this time was threaded cylindrical type.

\section{Conclusion}

We report a case of adult $\mathrm{BI}$ with $\mathrm{C} 1$ assimilation. After traction using a Halo vest, $\mathrm{C} 1-2$ facet distraction and fixation were performed in a "one-stage only" operation. A 6-mm threaded titanium cylindrical cage was very usefully for distraction of the C1-2 space and fix C1-2 facet joint, although Goel et al. used a distinct original spacer to distract C1-2 space.

Our surgical method achieved both fixation and decompression of the brainstem. Therefore, this modified version of Goel's method appears effective for treating BI.

\section{Conflicts of Interest Disclosure}

All authors have neither conflicts of interest nor financial interest in this work.

\section{References}

1) von Torklus D, Gehle W: [Upper cerebral vertebrae. Regional morphology, pathology and traumatology, practical x-ray atlas and taxonomy]. Fortschr Geb Rontgenstrahlen Nuklearmed Erganzungsbd 101: $1-156,1975$

2) Goel A, Desai KI, Muzumdar DP: Atlantoaxial fixation using plate and screw method: a report of 160 treated patients. Neurosurgery 51: 1351-1356; discussion 1356-1357, 2002

3) Goel A: Treatment of basilar invagination by atlantoaxial joint distraction and direct lateral mass fixation. J Neurosurg Spine 1: 281-286, 2004

4) Simsek S, Yigitkanli K, Belen D, Bavbek M: Halo traction in basilar invagination: technical case report. Surg Neurol 66: 311-314; discussion 314, 2006

5) Chamberlain WE: Basilar impression (platybasia): a bizarre developmental anomaly of the occipital bone and upper cervical spine with striking and misleading neurologic manifestations. Yale J Biol Med 11: 487-496, 1939

6) McRAE DL: Bony abnormalities in the region of the foramen magnum: correlation of the anatomic and neurologic findings. Acta radiol 40: 335-354, 1953

7) Goel A, Bhatjiwale M, Desai K: Basilar invagination: a study based on 190 surgically treated patients. J Neurosurg 88: 962-968, 1998

Corresponding author:

Shunji Asamoto, MD, PhD, Spine and Spinal Cord Center, Mita Hospital, International University of Health and Welfare, 1-4-3 Mita, Minato-ku, Tokyo 1088329, Japan.

$\triangle$ spine-asm@iuhw.ac.jp 